\title{
An Analysis of Heat Conduction Models for Nanofluids
}

João N. N. Quaresma , Emanuel N. Macêdo , Henrique M. da Fonseca , Helcio R. B. Orlande \& Renato M. Cotta

To cite this article: João N. N. Quaresma, Emanuel N. Macêdo, Henrique M. da Fonseca , Helcio R. B. Orlande \& Renato M. Cotta (2010) An Analysis of Heat Conduction Models for Nanofluids, Heat Transfer Engineering, 31:14, 1125-1136, DOI: 10.1080/01457631003689211

To link to this article: https://doi.org/10.1080/01457631003689211

Published online: 28 Oct 2010.

Submit your article to this journal $\square$

Џll Article views: 842

Citing articles: 10 View citing articles $[\pi$ 


\title{
An Analysis of Heat Conduction Models for Nanofluids
}

\author{
JOÃO N. N. QUARESMA, ${ }^{1}$ EMANUEL N. MACÊDO,${ }^{1}$ HENRIQUE M. DA \\ FONSECA, ${ }^{2}$ HELCIO R. B. ORLANDE, ${ }^{2}$ and RENATO M. COTTA ${ }^{2}$ \\ ${ }^{1}$ School of Chemical Engineering, Universidade Federal do Pará, UFPA Campus Universitário do Guamá, Belém, PA, Brazil \\ ${ }^{2}$ Mechanical Engineering Department-Politécnica/COPPE, Universidade Federal do Rio de Janeiro, Rio de Janeiro, RJ, Brazil
}

\begin{abstract}
The mechanism of heat transfer intensification recently brought about by nanofluids is analyzed in this article, in the light of the non-Fourier dual-phase-lagging heat conduction model. The physical problem involves an annular geometry filled with a nanofluid, such as typically used for measurements of the thermal conductivity with Blackwell's line heat source probe. The mathematical formulation for this problem is analytically solved with the classical integral transform technique, thus providing benchmark results for the temperature predicted with the dual-phase-lagging model. Different test cases are examined in this work, involving nanofluids and probe sizes of practical interest. The effects of the relaxation times on the temperature at the surface of the probe are also examined. The results obtained with the dualphase-lagging model are critically compared to those obtained with the classical parabolic model, showing that the increase in the thermal conductivity of nanofluids measured with the line heat source probe cannot be attributed to hyperbolic effects.
\end{abstract}

\section{INTRODUCTION}

The constitutive equation that classically relates the heat flux vector to the temperature gradient is Fourier's law, which considers an infinite speed of propagation of heat in the medium. Despite this unacceptable assumption, Fourier's law provides accurate results for most practical engineering applications. However, in applications involving small scales of time and space, the use of other constitutive equations, such as those independently derived by Cattaneo [1] and Vernotte [2], may be required. Such models take into account a lag between the heat flux vector and the temperature gradient, resulting in a hyperbolic model for heat conduction [1-13].

Thermal conductivity of fluids plays a vital role in the development of energy-efficient heat transfer equipment. However, traditional fluids used in those equipments have low thermal

The authors acknowledge the financial support provided by CNPq for the postdoctoral fellowship of Professor J. N. N. Quaresma at the Laboratory of Heat Transmission and Technology of the Mechanical Engineering Department of COPPE/UFRJ. This work was partially sponsored by CAPES and FAPERJ, with major financial support provided by Petrobras S.A.

Address correspondence to Professor Helcio R. B. Orlande, Mechanical Engineering Department-Politécnica/COPPE, Universidade Federal do Rio de Janeiro, UFRJ, Cx. Postal 68503-Cidade Universitária, 21941-972, Rio de Janeiro, RJ, Brazil. E-mail: helcio@mecanica.coppe.ufrj.br conductivity [14]. On the other hand, metals in the solid form have thermal conductivity larger by orders of magnitude than those of fluids. For example, the thermal conductivity of copper at room temperature is about 700 times larger than that of water and around 3000 times larger than that of engine oil. Therefore, fluids containing suspended solid metallic particles were expected to display significantly enhanced thermal conductivities relative to conventional heat transfer fluids. Numerous theoretical and experimental studies of the effective thermal conductivity of dispersions containing particles have been conducted since Maxwell's theoretical work on the subject was published more than 100 years ago $[14,15]$. However, early studies of the thermal conductivity of suspensions have been confined to those containing particles with sizes of the order of millimeters or micrometers. In fact, conventional micrometersized particles cannot be used in practical heat-transfer equipment because of severe clogging and sedimentation problems. In addition, recent miniaturization, leading to the increasing practical utilization of microchannels and microreactors, also imposed a restriction on the use of micrometer-sized particles [14, 16-18].

Modern nanotechnology provides great opportunities to process and produce materials with average sizes below $50 \mathrm{~nm}$ [14, 16-18]. Recognizing an opportunity to apply this emerging nanotechnology to established thermal energy engineering, 
Choi and coworkers [14, 16-18] proposed that nanometer-sized metallic particles be suspended in industrial heat transfer fluids, such as water or ethylene glycol, to produce a new class of engineered fluids referred to as nanofluids. Experiments with nanofluids have indicated significant increases in thermal conductivity, as compared to base liquids without nanoparticles or with larger suspended particles [14, 16-23]. Generally, the observed increase in thermal conductivity of nanofluids was substantially larger than that predicted with the available theory. On the other hand, some studies reported that such increase in the thermal conductivity could not be detected with optical experimental methods [24, 25]. In this context, such a fact resulted in a search for physical phenomena not accounted for in the theoretical predictions for suspensions of micrometer-sized or larger particles, which included Brownian motion, liquid layering, ballistic mechanisms, thermophoresis, aggregation of nanoparticles into clusters, etc. [16-26]. Due to previous experimental observations that non-Fourier effects are significant at small time and space scales [1-11], hyperbolic heat conduction models were naturally suggested to explain the heat transfer enhancement in nanofluids [12, 13]. It was proposed $[12,13]$ that enhanced heat transfer in nanofluids was caused by a heat transfer mechanism modeled in terms of the dual-phase-lagging constitutive equation $[5,7]$.

In this article we revisit the works of references [12] and [13] and apply the dual-phase-lagging model to a one-dimensional heat conduction problem in cylindrical coordinates. The geometry examined here is that typically used for the measurements of thermal conductivity of nanofluids with Blackwell's heat source probe [27]. Such a technique consists of a line heat source, usually taken in the form of a heating wire, which is placed inside the material with unknown properties. For large times, the temperature variation of the heat source is shown to be linear with respect to the logarithm of time, so that the thermal conductivity can be computed from the slope of such linear variation. The temperature variation of the heat source can be measured through the variation of the heating wire electrical conductivity. Alternatively, the heating wire and a temperature sensor, such as a thermocouple or a PT-100, can be encapsulated in a metallic needle that is inserted into the medium with unknown thermal conductivity [27-31]. Commercial probes are generally based on this last construction arrangement [31].

The heat conduction problem under examination is solved analytically by using the classical integral transform technique (CITT) $[32,33]$. The controlled accuracy and analytical nature of the solution technique developed in this work allow for the computation of benchmark results for the temperature variation of the probe, based on the dual-phase-lagging model. Numerical results are presented in this article for typical configurations of probes and nanofluids, as well as for different values of relaxation times, which result on hyperbolic effects with distinct intensities. Such results are critically compared to the classical parabolic heat conduction model, as well as to Blackwell's largetime solution.

\section{PROBLEM FORMULATION}

The analysis considered here is similar to that examined in references [12] and [13] and involves a dual-phase-lagging model (DPLM) [5, 7]. In such a model, a finite speed of heat propagation in the medium is taken into account through a delay time for the establishment of the heat flux, $\tau_{q}$, and a lag between the heat flux vector and the temperature gradient, $\tau_{T}$. The constitutive equation relating the heat conduction flux vector and the temperature gradient in the dual-phase-lagging model is given by $[1-7,12,13]$ :

$$
\mathbf{q}+\tau_{q} \frac{\partial \mathbf{q}}{\partial t}=-K\left[\nabla T_{f}+\tau_{T} \frac{\partial\left(\nabla T_{f}\right)}{\partial t}\right]
$$

where $\mathbf{q}$ is the heat flux vector and $K$ is the effective thermal conductivity of the medium.

The energy conservation equation for a purely conducting medium, considered in this work as a nanofluid, is written as

$$
\left(C_{s}+C_{f}\right) \frac{\partial T_{f}}{\partial t}=-\nabla \cdot \mathbf{q}
$$

where $C_{s}$ is the volumetric heat capacity of the nanoparticles and $C_{f}$ is the volumetric heat capacity of the base fluid.

The substitution of Eq. (1) into the energy conservation equation (2) results in:

$$
\tau_{q} \frac{\partial^{2} T_{f}}{\partial t^{2}}+\frac{\partial T_{f}}{\partial t}=\alpha \nabla^{2} T_{f}+\alpha \tau_{T} \frac{\partial\left(\nabla^{2} T_{f}\right)}{\partial t}
$$

with the effective thermal diffusivity given by:

$$
\alpha=\frac{K}{C_{s}+C_{f}}
$$

Therefore, the use of the constitutive Eq. (1) together with the energy conservation Eq. (2) leads to a hyperbolic heat conduction model given by Eq. (3).

Equation (3) can be similarly obtained by considering that the nanoparticles and the base fluid are not in local thermal equilibrium. In this case, the energy conservation equation can be written separately for the nanoparticles and the base fluid, respectively, in the following form:

$C_{s} \frac{\partial T_{s}}{\partial t}=h\left(T_{f}-T_{s}\right) ; C_{f} \frac{\partial T_{f}}{\partial t}=K \nabla^{2} T_{f}-h\left(T_{f}-T_{s}\right)$

where $h\left(\mathrm{~W} / \mathrm{m}^{30} \mathrm{C}\right)$ is a heat transfer coefficient between the fluid and the nanoparticles. Note that it was considered a lumped formulation for the nanoparticles, given by Eq. (5).

Then, by substituting $T_{s}$ from Eq. (5) into Eq. (6), one obtains:

$$
\begin{aligned}
& \frac{C_{s} C_{f}}{h\left(C_{s}+C_{f}\right)} \frac{\partial^{2} T_{f}}{\partial t^{2}}+\frac{\partial T_{f}}{\partial t} \\
& =\frac{K}{\left(C_{s}+C_{f}\right)} \nabla^{2} T_{f}+\frac{K}{\left(C_{s}+C_{f}\right)} \frac{C_{s}}{h} \frac{\partial\left(\nabla^{2} T_{f}\right)}{\partial t}
\end{aligned}
$$


A comparison of Eqs. (3) and (7) reveals the definition of the effective thermal diffusivity given by Eq. (4), as well of the relaxation times given by:

$$
\tau_{q}=\frac{C_{s} C_{f}}{h\left(C_{s}+C_{f}\right)} ; \quad \tau_{T}=\frac{C_{s}}{h}
$$

Therefore,

$$
\tau_{T}=\tau_{q} \frac{C_{s}+C_{f}}{C_{f}}
$$

or, alternatively,

$$
\frac{\tau_{T}}{\tau_{q}}=1+\frac{C_{s}}{C_{f}}>1
$$

Considering the case involving a line heat source probe of radius $a$ immersed in a cylindrical medium of radius $b$, Eq. (3) can be rewritten as

$\tau_{q} \frac{\partial^{2} T(r, t)}{\partial t^{2}}+\frac{\partial T(r, t)}{\partial t}=\alpha \frac{1}{r} \frac{\partial}{\partial r}\left\{r \frac{\partial}{\partial r}\left[T(r, t)+\tau_{T} \frac{\partial T(r, t)}{\partial t}\right]\right\}$,

$$
\text { in } a<r<b, \quad \text { for } t>0
$$

which is subjected to the following initial and boundary conditions:

$$
T(r, t)=T_{0} ; \quad \frac{\partial T(r, t)}{\partial t}=0 \quad \text { for } t=0, \quad \text { in } a<r<b
$$

$$
\begin{gathered}
-K \frac{\partial}{\partial r}\left[T(r, t)+\tau_{T} \frac{\partial T(r, t)}{\partial t}\right]=q_{0}+\tau_{q} \frac{\partial q_{0}}{\partial t} \\
\text { at } r=a, \quad \text { for } t>0
\end{gathered}
$$

$$
K \frac{\partial}{\partial r}\left[T(r, t)+\tau_{T} \frac{\partial T(r, t)}{\partial t}\right]=0 \quad \text { at } \quad r=b, \quad \text { for } \quad t>0
$$

where $q_{0}$ is the heat flux resulting from the electrical resistance inside the probe and $T_{0}$ is the initial temperature of the medium.

By defining the following dimensionless variables,

$$
\begin{aligned}
\theta(R, \tau) & =\frac{T(r, t)-T_{0}}{\left(q_{0} b / K\right)} ; R=\frac{r}{b} ; A=\frac{a}{b} ; \tau=\frac{\alpha t}{b^{2}} ; F o_{q}=\frac{\alpha \tau_{q}}{b^{2}} ; \\
F o_{T} & =\frac{\alpha \tau_{T}}{b^{2}} ; \beta=\frac{F o_{T}}{F o_{q}}
\end{aligned}
$$

the problem given by Eqs. (11a-e) can be rewritten in dimensionless form as:

$$
\begin{aligned}
& F o_{q} \frac{\partial^{2} \theta(R, \tau)}{\partial \tau^{2}}+\frac{\partial \theta(R, \tau)}{\partial \tau} \\
& =\frac{1}{R} \frac{\partial}{\partial R}\left\{R \frac{\partial}{\partial R}\left[\theta(R, \tau)+F o_{T} \frac{\partial \theta(R, \tau)}{\partial \tau}\right]\right\},
\end{aligned}
$$$$
\text { in } A<R<1, \tau>0
$$

$$
\theta(r, \tau)=0 ; \frac{\partial \theta(R, \tau)}{\partial \tau}=0 \quad \text { for } \quad \tau=0, \quad \text { in } A<R<1
$$

$$
-\frac{\partial}{\partial R}\left[\theta(R, \tau)+F o_{T} \frac{\partial \theta(R, \tau)}{\partial \tau}\right]=1 \quad \text { at } R=A, \quad \text { for } \tau>0
$$

$$
\frac{\partial}{\partial R}\left[\theta(R, \tau)+F o_{T} \frac{\partial \theta(R, \tau)}{\partial \tau}\right]=0 \text { at } \quad R=1, \text { for } \tau>0
$$

where it was considered that heat flux imposed by the probe is constant in time.

The classical parabolic heat conduction model, which utilizes Fourier's law as the constitutive equation that relates the heat conduction flux vector and the temperature gradient, can be directly obtained from Eqs. (13a)-(13e) by making the relaxation times, $\tau_{q}$ and $\tau_{T}$, equal to zero (Eq. (1)). In terms of the nonequilibrium model given by Eqs. (5) and (6), $\tau_{q} \rightarrow 0$ and $\tau_{T}$ $\rightarrow 0$ can be obtained with $h \rightarrow \infty$ (Eqs. (8a) and (8b)) - that is, the heat transfer coefficient between the fluid and the dispersed nanoparticles becomes very large and local thermal equilibrium is attained $\left(T_{f}=T_{s}\right)$.

\section{SOLUTION METHODOLOGY}

For the solution of the hyperbolic heat conduction problem given by Eqs. (13a)-(13e) we apply the classical integral transform technique (CITT) [32, 33]. A split-up procedure [32] is used in order to improve the convergence rate of the final series solution. Hence, the solution for the temperature field is written as:

$$
\theta(R, \tau)=\theta_{a v}(\tau)+\theta_{p}(R)+\phi(R, \tau)
$$

where $\theta_{a v}(\tau)$ is the average temperature in the medium, which is a priori obtained from Eqs. (13a) $-(13 \mathrm{e}) ; \theta_{p}(R)$ is a particular solution and $\phi(R, \tau)$ is the potential to be solved with the CITT.

The average temperature $\theta_{a v}(\tau)$ is defined as

$$
\theta_{a v}(\tau)=\frac{\int_{A}^{1} R \theta(R, \tau) d R}{\int_{A}^{1} R d R}=\frac{2}{1-A^{2}} \int_{A}^{1} R \theta(R, \tau) d R
$$

Now, in order to determine a solution for $\theta_{a v}(\tau)$, Eq. (13a) is multiplied by $\left[2 /\left(1-A^{2}\right)\right] R$ and integrated over the domain $[A, 1]$ in the $R$-direction. The definition given by Eq. (15) is then employed and the boundary conditions (13d, 13e) are used to yield

$$
F o_{q} \frac{d^{2} \theta_{a v}(\tau)}{d \tau^{2}}+\frac{d \theta_{a v}(\tau)}{d \tau}=\frac{2 A}{1-A^{2}}, \quad \text { for } \tau>0
$$

From the initial conditions (13b) and (13c) together with the definition (15), it results that

$$
\theta_{a v}(\tau)=0 ; \frac{d \theta_{a v}(\tau)}{d \tau}=0 \quad \text { for } \tau=0
$$

$(16 b, 16 c)$ 
Therefore, the solution for $\theta_{a v}(\tau)$ is obtained as

$$
\theta_{a v}(\tau)=\frac{2 A}{1-A^{2}}\left[\tau-F o_{q}\left(1-e^{-\tau / F o_{q}}\right)\right]
$$

Equation (14) is now introduced into Eqs. (13a)-(13e) and the problem for $\theta_{a v}(\tau)$ given by Eqs. (16a)-(16c) is used in order to obtain the following problems given by Eqs. (17a)-(17d) and Eqs. (18a-f), for $\theta_{p}(R)$ and $\phi(R, \tau)$, respectively:

$$
\begin{gathered}
\frac{1}{R} \frac{d}{d R}\left[R \frac{d \theta_{p}(R)}{d R}\right]=\frac{2 A}{1-A^{2}}, \quad \text { in } A<R<1 \quad \text { (17a) } \\
-\frac{d \theta_{p}(R)}{d R}=1 \quad \text { at } R=A ; \frac{d \theta_{p}(R)}{d R}=0 \quad \text { at } R=1(17 \mathrm{~b}, 17 \mathrm{c})
\end{gathered}
$$

with

$$
\int_{A}^{1} R \theta_{p}(R) d R=0
$$

and

$$
\begin{aligned}
& F o_{q} \frac{\partial^{2} \phi(R, \tau)}{\partial \tau^{2}}+\frac{\partial \phi(R, \tau)}{\partial \tau} \\
& =\frac{1}{R} \frac{\partial}{\partial R}\left\{R \frac{\partial}{\partial R}\left[\phi(R, \tau)+F o_{T} \frac{\partial \phi(R, \tau)}{\partial \tau}\right]\right\}, \\
& \quad \text { in } A<R<1, \quad \tau>0
\end{aligned}
$$

$\phi(R, \tau)=-\theta_{p}(R) ; \frac{\partial \phi(R, \tau)}{\partial \tau}=0 \quad$ for $\tau=0, \quad$ in $A<R<1$

$(18 \mathrm{~b}, 18 \mathrm{c})$

$$
-\frac{\partial}{\partial R}\left[\phi(R, \tau)+F o_{T} \frac{\partial \phi(R, \tau)}{\partial \tau}\right]=0 \text { at } R=A \text {, for } \tau>0
$$

$$
\frac{\partial}{\partial R}\left[\phi(R, \tau)+F o_{T} \frac{\partial \phi(R, \tau)}{\partial \tau}\right]=0 \text { at } R=1, \text { for } \tau>0
$$

with

$$
\int_{A}^{1} R \phi(R, \tau) d R=0
$$

The additional constraints given by Eqs. (17d) and (18f) are obtained by substituting Eq. (14) into the definition of the average temperature $\theta_{a v}(\tau)$ given by Eq. (15).

The integration of the problem given by Eqs. (17a)-(17d) can be readily performed in order to obtain the solution for the potential $\theta_{p}(R)$ in the form:

$$
\begin{aligned}
\theta_{p}(R)= & \frac{A}{1-A^{2}}\left[\frac{R^{2}}{2}-\ln (R)\right]-\frac{A}{4\left(1-A^{2}\right)^{2}} \\
& \times\left[4 A^{2} \ln (A)+\left(3+A^{2}\right)\left(1-A^{2}\right)\right]
\end{aligned}
$$

The homogeneous problem for the potential $\phi(R, \tau)$ is now solved with the classical integral transform technique (CITT) $[32,33]$. For this purpose, the following auxiliary eigenvalue problem is utilized, which shall provide the basis for the eigenfunction expansion of the potential $\phi(R, \tau)$ :

$$
\begin{gathered}
\frac{d}{d R}\left[R \frac{d \Gamma_{i}(R)}{d R}\right]+\beta_{i}^{2} R \Gamma_{i}(R)=0, \quad \text { in } \mathrm{A}<R<1(20 \mathrm{a}) \\
\frac{d \Gamma_{i}(R)}{d R}=0 \text { at } R=A ; \frac{d \Gamma_{i}(R)}{d R}=0 \text { at } R=1(20 \mathrm{~b}, 20 \mathrm{c})
\end{gathered}
$$

Equations $(20 \mathrm{a}-\mathrm{c})$ can be analytically solved to yield the eigenfunctions and the transcendental equation to compute the eigenvalues respectively as $[32,33]$ :

$$
\begin{gathered}
\Gamma_{i}(R)=J_{o}\left(\beta_{i} R\right) Y_{1}\left(\beta_{i}\right)-J_{1}\left(\beta_{i}\right) Y_{0}\left(\beta_{i} R\right) \\
J_{1}\left(\beta_{i} A\right) Y_{1}\left(\beta_{i}\right)-J_{1}\left(\beta_{i}\right) Y_{1}\left(\beta_{i} A\right)=0, \quad i=1,2,3, \ldots
\end{gathered}
$$

It can be shown that the eigenfunctions $\Gamma_{\mathrm{i}}(R)$ satisfy the following orthogonality property $[32,33]$ :

$$
\int_{A}^{1} R \Gamma_{i}(R) \Gamma_{j}(R) d R= \begin{cases}0, & i \neq j \\ N_{i}, & i=j\end{cases}
$$

where $N_{i}$ is the normalization integral given by:

$$
N_{i}=\frac{2}{\pi^{2}} \frac{J_{1}^{2}\left(\beta_{i} A\right)-J_{1}^{2}\left(\beta_{i}\right)}{\beta_{i}^{2} J_{1}^{2}\left(\beta_{i} A\right)}
$$

The auxiliary eigenvalue problem given by Eqs. (20a)-(20c) allows the definition of the following integral transform-inverse pair for the potential $\phi(R, \tau)$ :

$$
\begin{gathered}
\bar{\phi}_{i}(\tau)=\int_{A}^{1} R \tilde{\Gamma}_{i}(R) \phi(R, \tau) d R, \quad \text { transform } \\
\phi(R, \tau)=\sum_{i=1}^{\infty} \tilde{\Gamma}_{i}(R) \bar{\phi}_{i}(\tau), \quad \text { inverse }
\end{gathered}
$$

where

$$
\tilde{\Gamma}_{i}(R)=\Gamma_{i}(R) / \sqrt{N_{i}}
$$

After the definition of the integral transform-inverse pair with the auxiliary eigenvalue problem (20a)-(20c), the next step in the CITT is thus to accomplish the integral transformation of the original partial differential system given by Eqs. (18a)-(18e). For this purpose, Eq. (18a) and the initial conditions (18b) and (18c) are multiplied by $R \tilde{\Gamma}_{i}(R)$, integrated over the domain $[A, 1]$ in the $R$-direction, and the inverse formula given by Eq. (22b) is employed. After the appropriate manipulations, the following system of ordinary differential results, for the calculation of the transformed potentials $\bar{\phi}_{i}(\tau)$ :

$\frac{d^{2} \bar{\phi}_{i}(\tau)}{d \tau^{2}}+\frac{\left(1+F o_{T} \beta_{i}^{2}\right)}{F o_{q}} \frac{d \bar{\phi}_{i}(\tau)}{d \tau}+\frac{\beta_{i}^{2}}{F o_{q}} \bar{\phi}_{i}(\tau)=0, \quad$ for $\tau>0$ 


$$
\bar{\phi}_{i}(\tau)=\bar{f}_{i} ; \frac{d \bar{\phi}_{i}(\tau)}{d \tau}=0 \quad \text { for } \tau=0
$$

where

$$
\bar{f}_{i}=-\int_{A}^{1} R \tilde{\Gamma}_{i}(R) \theta_{p}(R) d R
$$

for $i=1,2,3, \ldots$

The infinite system of ordinary differential equations $(23 a-c)$ is uncoupled and can be readily solved to yield:

$$
\begin{gathered}
\bar{\phi}_{i}(\tau)=\frac{\bar{f}_{i}}{2} \exp \left[-\frac{\left(1+F o_{T} \beta_{i}^{2}\right)}{2 F o_{q}}\left(1-\sqrt{1-\frac{4 F o_{q} \beta_{i}^{2}}{\left(1+F o_{T} \beta_{i}^{2}\right)^{2}}}\right) \tau\right] \\
\times\left\{\left[1+\frac{1}{\sqrt{1-\frac{4 F o_{q} \beta_{i}^{2}}{\left(1+F o_{T} \beta_{i}^{2}\right)^{2}}}}\right]+\left[1-\frac{1}{\sqrt{1-\frac{4 F o_{q} \beta_{i}^{2}}{\left(1+F o_{T} \beta_{i}^{2}\right)^{2}}}}\right]\right. \\
\left.\quad \times \exp \left[-\frac{\left(1+F o_{T} \beta_{i}^{2}\right)}{F o_{q}} \sqrt{ } \sqrt{\frac{4 F o_{q} \beta_{i}^{2}}{\left(1+F o_{T} \beta_{i}^{2}\right)^{2}}}\right]\right\}
\end{gathered}
$$

By substituting Eq. (24) into the inverse formula (22b), the analytical solution for the homogeneous potential $\phi(R, \tau)$ is obtained as

$$
\begin{aligned}
& \phi(R, \tau)=\sum_{i=1}^{\infty} \frac{\bar{f}_{i}}{2} \tilde{\Gamma}_{i}(R) \\
& \times \exp \left[-\frac{\left(1+F o_{T} \beta_{i}^{2}\right)}{2 F o_{q}}\left(1-\sqrt{1-\frac{4 F o_{q} \beta_{i}^{2}}{\left(1+F o_{T} \beta_{i}^{2}\right)^{2}}}\right) \tau\right] \\
& \times\left\{\left[1+\frac{1}{\sqrt{1-\frac{4 F o_{q} \beta_{i}^{2}}{\left(1+F o_{T} \beta_{i}^{2}\right)^{2}}}}\right]+\left[1-\frac{1}{\sqrt{1-\frac{4 F o_{q} \beta_{i}^{2}}{\left(1+F o_{T} \beta_{i}^{2}\right)^{2}}}}\right]\right. \\
& \left.\times \exp \left[-\frac{\left(1+F o_{T} \beta_{i}^{2}\right)}{F o_{q}} \sqrt{1-\frac{4 F o_{q} \beta_{i}^{2}}{\left(1+F o_{T} \beta_{i}^{2}\right)^{2}}}\right]\right\}
\end{aligned}
$$

Finally, by substituting Eqs. (16d), (19), and (25) into Eq. (14), the solution for the dimensionless temperature field is obtained as

$$
\begin{aligned}
& \theta(R, \tau)=\frac{2 A}{1-A^{2}}\left[\tau-F o_{q}\left(1-e^{-\tau / F o_{q}}\right)\right] \\
& +\frac{A}{1-A^{2}}\left[\frac{R^{2}}{2}-\ln (R)\right]-\frac{A}{4\left(1-A^{2}\right)^{2}} \\
& \quad \times\left[4 A^{2} \ln (A)+\left(3+A^{2}\right)\left(1-A^{2}\right)\right]+\sum_{i=1}^{\infty} \frac{\bar{f}_{i}}{2} \tilde{\Gamma}_{i}(R)
\end{aligned}
$$

heat transfer engineering

$$
\begin{aligned}
& \times \exp \left[-\frac{\left(1+F o_{T} \beta_{i}^{2}\right)}{2 F o_{q}}\left(1-\sqrt{\left.1-\frac{4 F o_{q} \beta_{i}^{2}}{\left(1+F o_{T} \beta_{i}^{2}\right)^{2}}\right)}\right]\right. \\
& \times\left\{\left[1+\frac{1}{\sqrt{1-\frac{4 F o_{q} \beta_{i}^{2}}{\left(1+F o_{T} \beta_{i}^{2}\right)^{2}}}}\right]+\left[1-\frac{1}{\sqrt{1-\frac{4 F o_{q} \beta_{i}^{2}}{\left(1+F o_{T} \beta_{i}^{2}\right)^{2}}}}\right]\right. \\
& \times \exp \left[-\frac{\left(1+F o_{T} \beta_{i}^{2}\right)}{F o_{q}} \sqrt{\left.\left.1-\frac{4 F o_{q} \beta_{i}^{2}}{\left(1+F o_{T} \beta_{i}^{2}\right)^{2}} \tau\right]\right\}}\right.
\end{aligned}
$$

The CITT is also applied in order to obtain the solution for the classical parabolic problem, based on Fouriers's law. In this case, the analytical solution is given by:

$$
\begin{aligned}
\theta(R, \tau)= & \frac{2 A}{1-A^{2}} \tau+\frac{A}{1-A^{2}}\left[\frac{R^{2}}{2}-\ln (R)\right] \\
& -\frac{A}{4\left(1-A^{2}\right)^{2}}\left[4 A^{2} \ln (A)+\left(3+A^{2}\right)\left(1-A^{2}\right)\right] \\
& +\sum_{i=1}^{\infty} \bar{f}_{i} \tilde{\Gamma}_{i}(R) \mathrm{e}^{-\beta_{i}^{2} \tau}
\end{aligned}
$$

where the eigenquantities that appear in Eq. (27) are the same as those defined earlier for the solution of the problem for the potential $\phi(R, \tau)$.

For large times, Blackwell [27] derived an asymptotic solution for the temperature variation at the surface of the line heat source probe in the parabolic problem, which is shown to be linear with respect to the logarithm of time. Such solution is convenient for the measurement of the thermal conductivity of the medium surrounding the probe, which can be computed from the slope of the temperature variation [27-31]. Blackwell's solution [27], in terms of the dimensionless variables given by Eqs. (12a), is

$$
\theta_{a}(\tau)=\frac{1}{2} A \ln \tau+\frac{1}{2} A\left[\ln \left(\frac{4}{A^{2}}\right)-y\right]
$$

where $y=0.5772156649$ is Euler's constant. We note in Eq. (28) that, in dimensionless terms, the slope of $\theta_{a}(\tau) \times \ln \tau$ is equal to A/2.

\section{RESULTS AND DISCUSSION}

In this session we present numerical results for the dimensionless temperature variation at the surface of the probe, that is, at $R=A$, obtained with the hyperbolic heat conduction model given by Eq. (26). Such results are compared to those obtained with the classical parabolic problem given by Eq. (27), as well as to those obtained with Blackwell's model given by Eq. (28). Such analytical solutions were implemented under the Visual Fortran platform.

vol. 31 no. 142010 
Table 1 Test cases examined

\begin{tabular}{|c|c|c|c|c|c|}
\hline \multirow{2}{*}{$\begin{array}{l}\text { Test } \\
\text { case }\end{array}$} & \multirow[b]{2}{*}{ Nanofluid } & \multicolumn{2}{|c|}{ Dimensions } & \multirow[b]{2}{*}{ A } & \multirow[b]{2}{*}{$\beta$} \\
\hline & & $a(m)$ & $b(m)$ & & \\
\hline $\mathrm{a}$ & Alumina in water & $5 \times 10^{-5}$ & 0.025 & $2 \times 10^{-3}$ & 1.71 \\
\hline$b$ & Alumina in water & $7.5 \times 10^{-4}$ & 0.05 & $1.5 \times 10^{-2}$ & 1.71 \\
\hline $\mathrm{c}$ & Copper in ethylene glycol & $5 \times 10^{-5}$ & 0.025 & $2 \times 10^{-3}$ & 2.29 \\
\hline d & Copper in ethylene glycol & $7.5 \times 10^{-4}$ & 0.05 & $1.5 \times 10^{-2}$ & 2.29 \\
\hline
\end{tabular}

Different test cases were examined in this work, involving different configurations of probe diameters and nanofluids. With respect to the nanofluids, the following ones were considered in the analysis: (i) alumina nanoparticles in water $(K=0.257$ $\mathrm{W} / \mathrm{mK}, C_{s}=3.430 \times 10^{6} \mathrm{~J} / \mathrm{m}^{3} \mathrm{~K}, C_{f}=2.649 \times 10^{6} \mathrm{~J} / \mathrm{m}^{3}-\mathrm{K}$, $\beta=1.71)$ and (ii) copper nanoparticles in ethylene glycol $(K=$ $0.627 \mathrm{~W} / \mathrm{m}-\mathrm{K}, C_{s}=2.964 \times 10^{6} \mathrm{~J} / \mathrm{m}^{3}-\mathrm{K}, C_{f}=4.183 \times 10^{6}$ $\mathrm{J} / \mathrm{m}^{3}-\mathrm{K}, \beta=2.29$ ). With respect to the probe geometry, it was considered to be made of a thin resistance wire with diameter $a=5 \times 10^{-5} \mathrm{~m}$ inserted into a medium with outer diameter $b=0.025 \mathrm{~m}$, so that $A=2 \times 10^{-3}$, such as in [12] and [13]. Also examined was another probe with diameter $a=7.5 \times$ $10^{-4} \mathrm{~m}$, typical of those commercially available [31]. In this case, the medium was considered with an outer diameter $b=$ $0.05 \mathrm{~m}$, so that $A=1.5 \times 10^{-2}$. Table 1 summarizes the test cases examined.

Figure 1 illustrates the convergence behavior of the temperature at the probe surface, obtained with different truncation orders $(N T)$ for the series solution in Eq. (26), for $A=2 \times 10^{-3}$, $F o_{q}=1 \times 10^{-5}$, and $\beta=2.29$ (test case c). This figure shows that for small dimensionless times $\left(\tau \leq 10^{-5}\right)$, convergence at

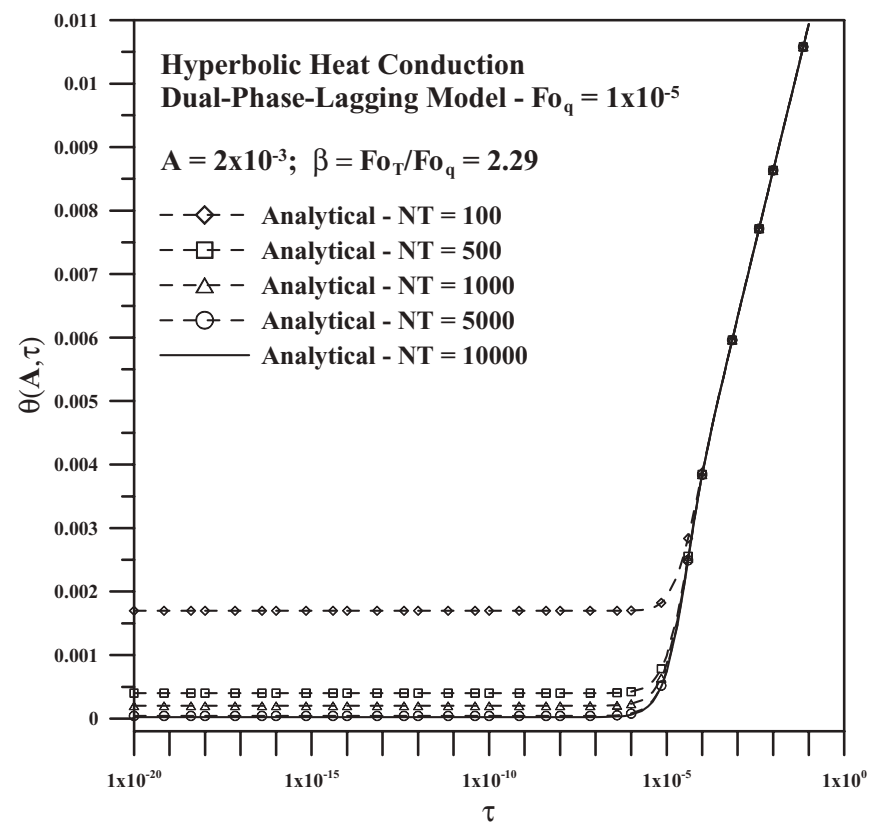

Figure 1 Convergence behavior of the analytical solution for $A=2 \times 10^{-3}$, $F o_{q}=1 \times 10^{-5}$, and $\beta=2.29$.

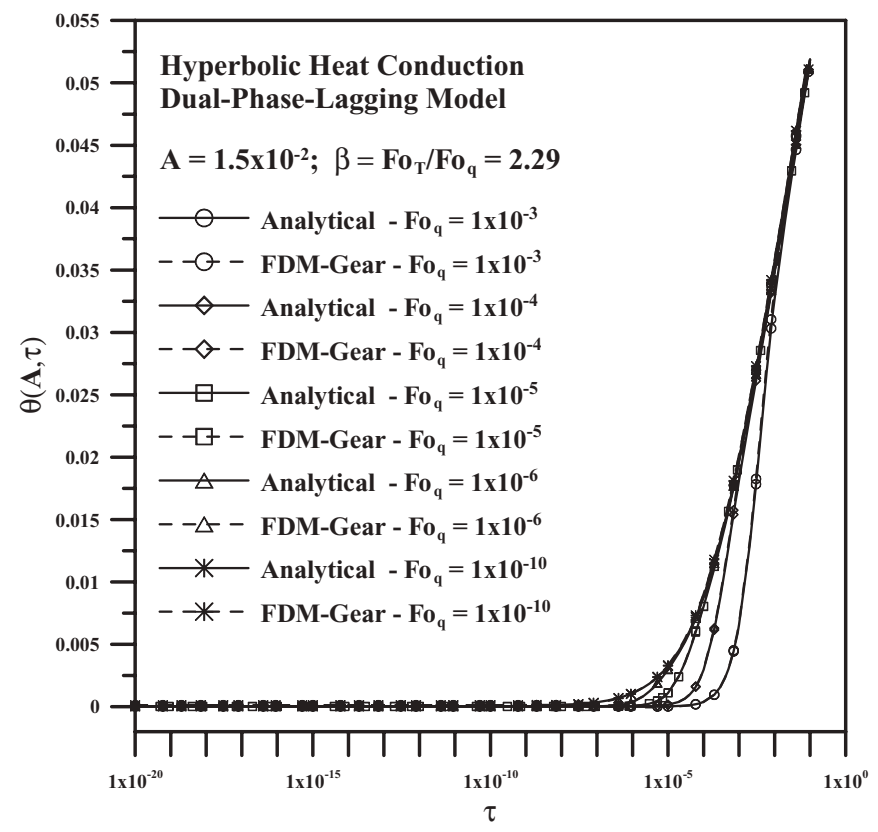

Figure 2 Comparison of analytical solution and finite-difference method solution using Gear's method (referred to as FDM-Gear) for $A=1.5 \times 10^{-2}$ and $\beta=2.29$.

the graphic scale is obtained with $5000 \leq N T \leq 10000$. On the other hand, for larger dimensionless times the convergence is reached with approximately 500 terms in the series solution. The computation time in a Pentium Intel Dual E2160 1.8-GHz computer was of the order of 9.7 minutes, for $N T=10000$. For the results presented next, $N T=10000$ was used.

In order to validate the analytical solution just presented, we compared its results with those obtained numerically with finite

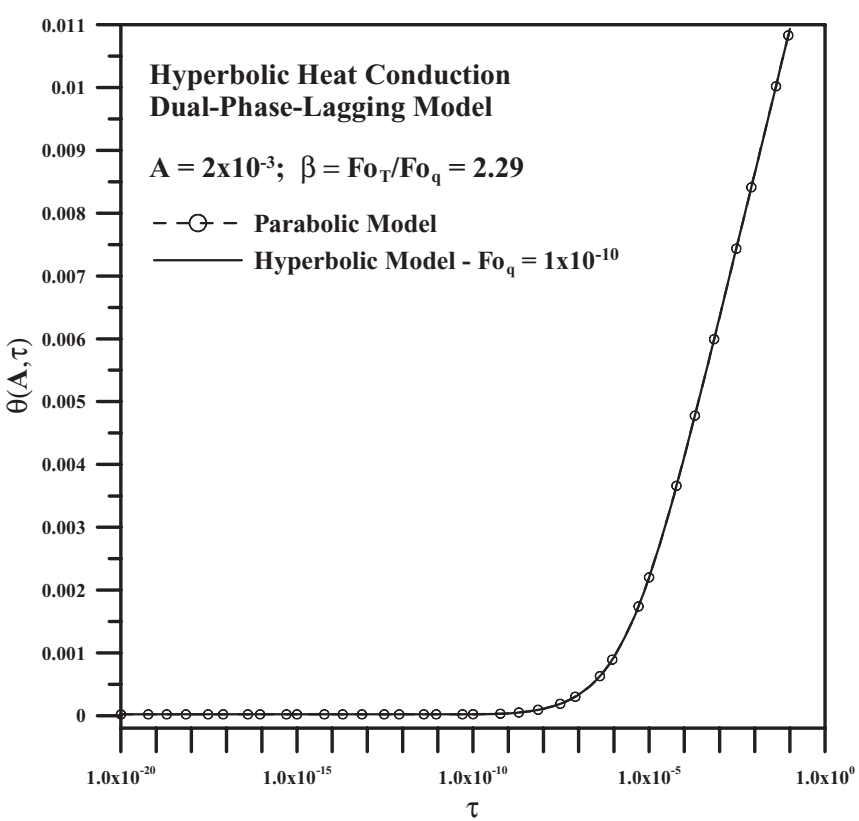

Figure 3 Comparison of the hyperbolic $\left(F o_{q}=10^{-10}\right)$ and parabolic solutions for $A=2 \times 10^{-3}$ and $\beta=2.29$.

vol. 31 no. 142010 

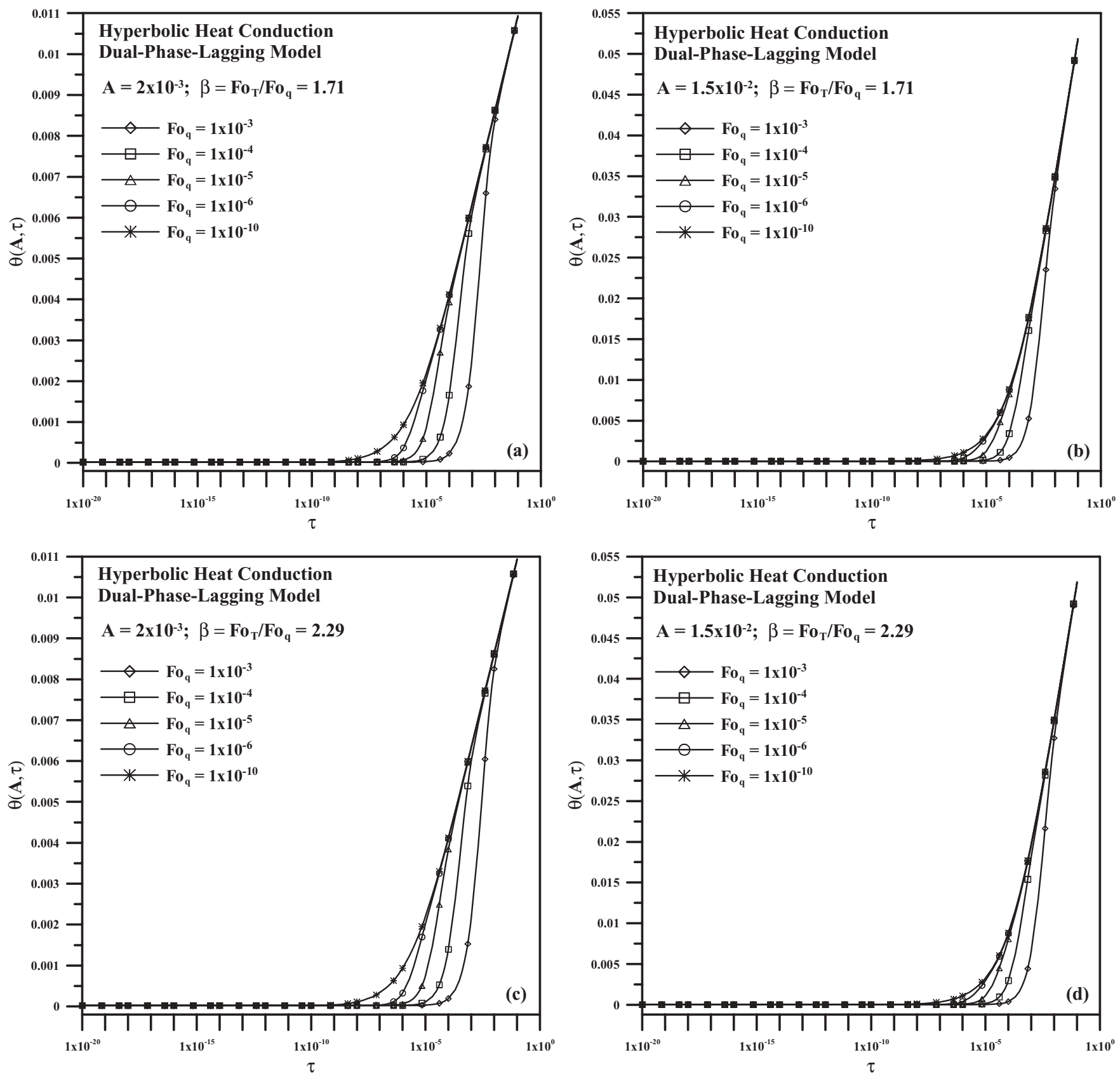

Figure 4 Temperature variation at the probe surface for test cases: (a) $A=2 \times 10^{-3}$ and $\beta=1.71$; (b) $A=1.5 \times 10^{-2}$ and $\beta=1.71$; (c) $A=2 \times 10^{-3}$ and $\beta=$ 2.29 ; (d) $A=1.5 \times 10^{-2}$ and $\beta=2.29$.

differences. In this case, the problem given by Eqs. (13a)-(13e) was discretized in the radial direction with second-order differences and the resulting system of ordinary differential equations was integrated in time with Gear's method. Figure 2 shows a comparison of the results obtained with the analytical solution against those obtained with the finite-difference method solution (referred to in Figure 2 by FDM-Gear) for test case d ( $A=$ $1.5 \times 10^{-2}, \beta=2.29$ ) and different values of $F_{q}$. The agreement between such solutions is excellent, thus validating the numerical code here developed. The finite-difference solution was obtained with 2000 nodes in the spatial grid.
Figure 3 presents a comparison of the hyperbolic and parabolic solutions given by Eqs. (26) and (27), respectively, for test case c $\left(A=2 \times 10^{-3}\right.$ and $\left.\beta=2.29\right)$ and $F o_{q}=1 \times$ $10^{-10}$. We note in this figure that for such a small value of $\mathrm{Fo}_{q}$ the hyperbolic model behaves exactly as the parabolic one, that is, non-Fourier effects are negligible. Such was also the case for other values of $A$ and $\beta$ examined in this article.

We now examine the non-Fourier effects of the probe surface temperature variation, for $\mathrm{Fo}_{q}$ ranging from $10^{-3}$ to $10^{-10}$. The results obtained for the different test cases described in Table 1 are presented in Figure $4 \mathrm{a}-\mathrm{d}$. These figures show that 

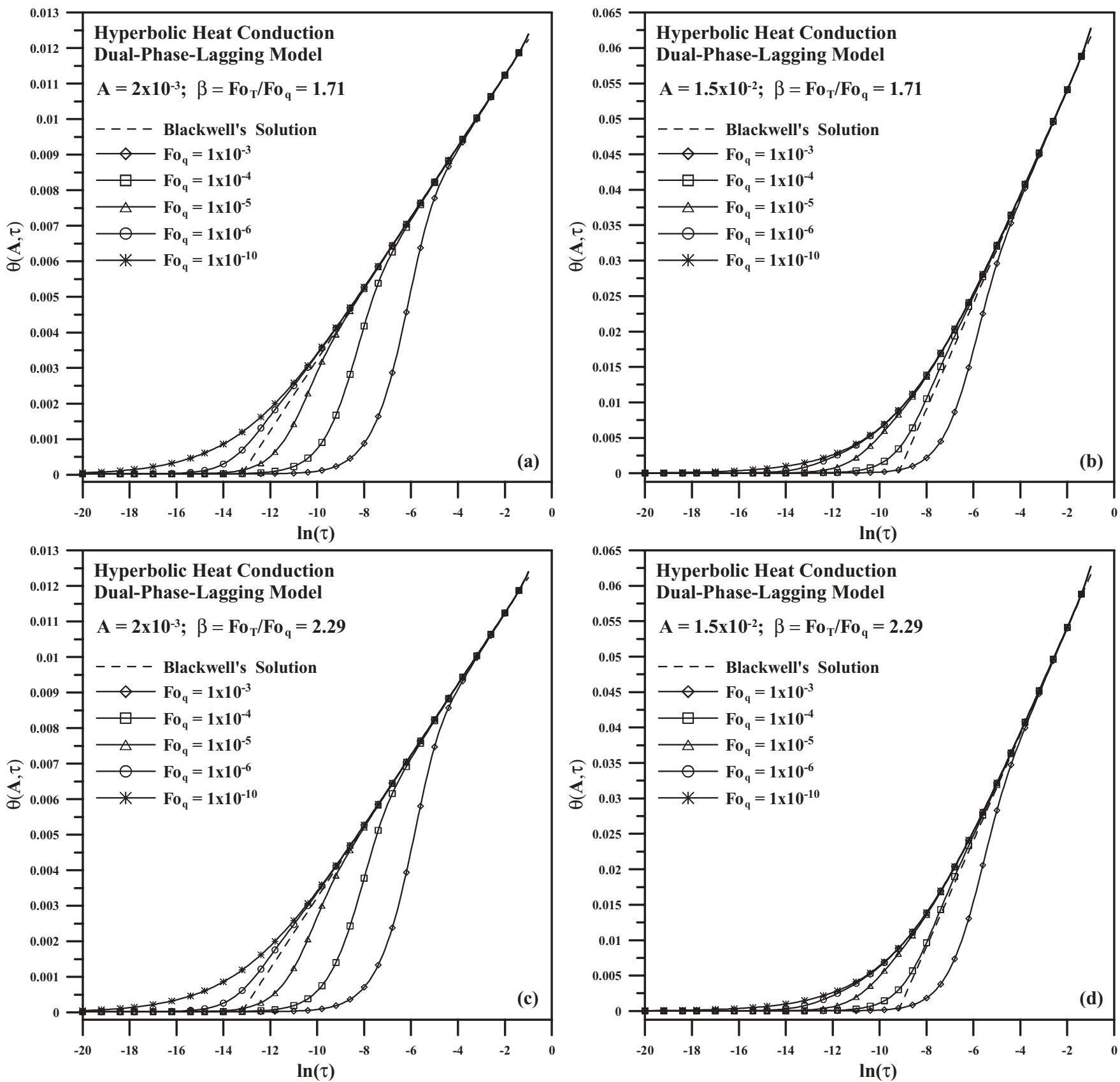

Figure 5 Comparison of hyperbolic and Blackwell's [27] solutions for test cases: (a) $A=2 \times 10^{-3}$ and $\beta=1.71$; (b) $A=1.5 \times 10^{-2}$ and $\beta=1.71$; (c) $A=2 \times$ $10^{-3}$ and $\beta=2.29$; (d) $A=1.5 \times 10^{-2}$ and $\beta=2.29$.

non-Fourier effects are only noticeable for very small times; as time increases, the temperature variations gradually tend to the parabolic one. In fact, even for an extremely large value of $\mathrm{Fo}_{q}$ such as $10^{-3}$, the non-Fourier effects vanish for $\tau>$ $10^{-2}$. At small times, non-Fourier effects are more pronounced for smaller diameters $A$. On the other hand, the choice of the nanofluid does not seem to affect significantly the temperature behavior. Similar conclusions can be obtained by comparing the hyperbolic solution with the asymptotic one de- veloped by Blackwell for the parabolic formulation, as depicted in Figure 5a-d.

The results presented in Figures $4 a-d$ and $5 a-d$ permit to examine the suitability of the hyperbolic formulation to the actual heat conduction problem in nanofluids, during thermal conductivity measurements with the line heat source probe. For this analysis, we bring into picture the heat transfer coefficient between the base fluid and the particles considered in the thermal nonequilibrium model given by Eqs. (5) and (6), which results 
Table 2 Dimensional times corresponding to $\tau=10^{-4}$

\begin{tabular}{lcc}
\hline & \multicolumn{2}{c}{ Time } \\
\cline { 2 - 3 } Test case & $\tau$ & $t(\mathrm{~s})$ \\
\hline $\mathrm{a}$ & $10^{-4}$ & 0.7 \\
$\mathrm{~b}$ & $10^{-4}$ & 2.9 \\
$\mathrm{c}$ & $10^{-4}$ & 1.5 \\
$\mathrm{~d}$ & $10^{-4}$ & 5.9 \\
\hline
\end{tabular}

in the hyperbolic formulation addressed in this article. Note in these equations that such heat transfer coefficient is defined in volumetric terms, but can be easily converted to the usual definition of the heat transfer coefficient by using the nanoparticle's volume to surface area ratio. Figure 6 presents the heat transfer coefficient between the base fluid and the particles for different values of $\mathrm{Fo}_{\mathrm{q}}$, and for spherical particles of different diameters. Only test cases a and c are examined in this figure, since they present more significant non-Fourier effects (Figures $4 \mathrm{a}-\mathrm{d}$ and $5 \mathrm{a}-\mathrm{d}$ ). By considering a threshold value for the heat transfer coefficient, it is possible to establish the maximum expected value of $\mathrm{Fo}_{q}$ for which the system behaves hyperbolically. If we assume such a threshold value as $1 \mathrm{~W} / \mathrm{m}^{2}-\mathrm{K}$, which is indeed extremely small in macroscopic means, we notice in Figure 6 that $\mathrm{Fo}_{q}$ is actually smaller than $10^{-5}$. Figure $4 \mathrm{a}-\mathrm{d}$, shows that for $\mathrm{Fo}_{q}=10^{-5}$, non-Fourier effects are negligible for $\tau>10^{-4}$. Table 2 gives the physical times equivalent to $\tau=10^{-4}$ for the four test cases addressed in this work. Notice in this table that non-Fourier effects would have disappeared for times much smaller than those typically considered for the measurement of the thermal conductivity with Blackwell's solution for the line heat source probe [27-31]. Indeed, notice in Figure 5, a-d,

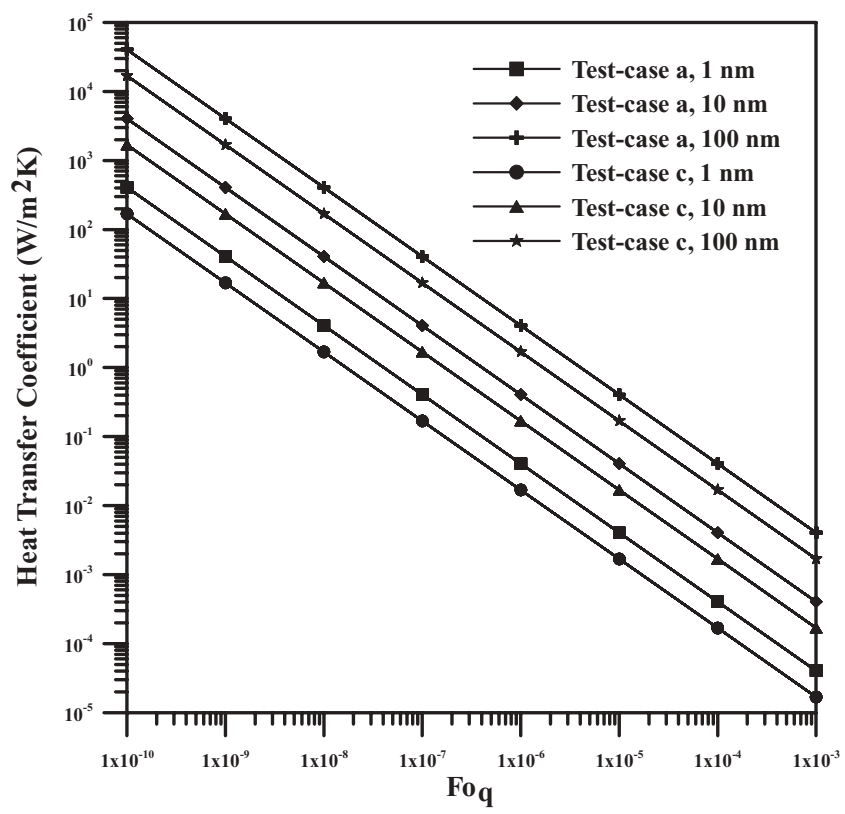

Figure 6 Heat transfer coefficient for different nanoparticle diameters. that Blackwell's solution would not be considered appropriate for the measurement of the thermal conductivity for $\tau<1.2$ $\times 10^{-4}(\ln \tau=-9)$ for test cases a and $\mathrm{c}$, and for $\tau<2.5 \times$ $10^{-3}(\ln \tau=-6)$ for test cases $b$ and $d$. In other words, the increase generally detected with the line heat source probe for the thermal conductivity of nanofluids cannot be attributed to the non-Fourier heat transfer mechanisms examined earlier. In fact, recent theoretical predictions corroborate our findings and demonstrate that nanoparticles and the base fluid are in thermal equilibrium in nanofluids $[23,34]$.

\section{CONCLUSIONS}

In this article we presented an analytical solution based on the classical integral transform technique for the dual-phase-lagging heat conduction model. The physical problem examined was representative of that used for the measurement of thermal conductivity with the line heat source probe. Results were obtained for the temperature variation at the probe surface, for different combinations of nanofluids and probe diameters. Such results were compared to those obtained with the classical parabolic heat conduction model based on Fourier's law, as well as to the asymptotic solution proposed by Blackwell [27] for the line heat source probe.

The foregoing analysis reveals that non-Fourier effects are significant only for very small times, generally in the range where Blackwell's solution is not valid for the measurement of thermal conductivity. Therefore, the increase detected with the line heat source probe for the thermal conductivity of nanofluids cannot be attributed to the non-Fourier heat transfer mechanisms addressed in this article.

\section{NOMENCLATURE}

a probe radius

A dimensionless probe radius

$b \quad$ radius of the cylindrical medium

$C_{f} \quad$ volumetric thermal capacity of the base fluid

$C_{s} \quad$ volumetric thermal capacity of the nanoparticles

$\bar{f}_{i} \quad$ transformed initial condition

$\mathrm{Fo}_{q}$ dimensionless relaxation time associated with the heat flux

$F_{T}$ dimensionless relaxation time associated with the temperature gradient

$h \quad$ heat transfer coefficient

$K \quad$ effective thermal conductivity of the nanofluid

$N_{i} \quad$ normalization integral

$N T$ truncation order in the summations

$q$ heat flux vector

$q_{0} \quad$ heat flux at the surface of the probe

$r$ radial variable

$R \quad$ dimensionless radial variable

$t$ time variable

$\mathrm{T}$ temperature 
$T_{f} \quad$ temperature of the base fluid

$T_{s} \quad$ temperature of the nanoparticles

$T_{0} \quad$ initial temperature

y Euler's constant

\section{Greek Symbols}

$\alpha \quad$ thermal diffusivity of the nanofluid

$\beta \quad$ ratio of relaxation times

$\beta_{i} \quad$ eigenvalues

$\Gamma_{i} \quad$ eigenfunctions

$\tilde{\Gamma}_{i} \quad$ normalized eigenfunctions

$\theta$ dimensionless temperature

$\theta_{a}$ dimensionless temperature from Blackwell's solution

$\theta_{a v}$ dimensionless average temperature

$\theta_{p} \quad$ particular solution for the dimensionless temperature field

$\rho$ density

$\tau$ dimensionless time variable

$\tau_{q} \quad$ relaxation time associated with the heat flux

$\tau_{T}$ relaxation time associated with the temperature gradient

$\phi \quad$ homogeneous solution for the dimensionless temperature field

$\bar{\phi}_{i} \quad$ transformed potentials

\section{Subscripts}

$i \quad$ order of the eigenvalue problem

$f$ relative to the base fluid

$s$ relative to the nanoparticles

\section{Superscripts}

- $\quad$ integral transformed quantities

$\sim$ normalized eigenfunctions

\section{REFERENCES}

[1] Cattaneo, C., A Form of Heat Conduction Equation Which Eliminates the Paradox of Instantaneous Propagation, Comptes Rendus Hebdomadaires des Séances de l'Académie des Sciences, vol. 247, pp. 431-433, 1958.

[2] Vernotte, P., Les Paradoxes de la Théorie Continue de l'Équation de la Chaleur, Comptes Rendus Hebdomadaires des Séances de l'Académie des Sciences, vol. 246, pp. 3154-3155, 1958.

[3] Joseph, D. D., and Preziosi, L., Heat Waves, Reviews of Modern Physics, vol. 61, pp. 41-73, 1989.

[4] Joseph, D. D., and Preziosi, L., Addendum to the Paper "Heat Waves," Reviews of Modern Physics, vol. 62, pp. 375-391, 1990.

[5] Tzou, D. Y., Macro-to-Microscale Heat Transfer: The Lagging Behavior, Taylor \& Francis, Washington, DC, 1997.

[6] Orlande, H. R. B., and Özisik, M. N., Simultaneous Estimations of Thermal Diffusivity and Relaxation Time With a Hyperbolic Heat Conduction Model, Proceedings of the Tenth International Heat Transfer Conference, Brighton, UK, vol. 6, pp. 403-408, 1994.

[7] Orlande, H. R. B., Özisik, M. N., and Tzou, D. Y., Inverse Analysis for Estimating the Electron-Phonon Coupling Factor in Thin Metal Films, Journal of Applied Physics, vol. 78, pp. 1843-1849, 1995.

[8] Chen, G., Thermal Conductivity and Ballistic-Phonon Transport in the Cross-Plane Direction of Superlattices, Physical Review B, vol. 57, pp. 14958-14973, 1998.

[9] Chen, G., Ballistic-Diffusive Heat Conduction Equations, Physical Review Letters, vol. 86, pp. 2297-2300, 2001.

[10] Yang, B., and Chen, G., Partially Coherent Phonon Heat Conduction in Superlattices, Physical Review B, vol. 67, pp. 1195311-1-1195311-4, 2003.

[11] Yang, R. G., and Chen, G., Thermal Conductivity Modeling of Periodic Two-Dimensional Nanocomposites, Physical Review B, vol. 69, pp. 195316-1-195316-10, 2004.

[12] Vadasz, J. J., Govender, S., and Vadasz, P., Heat Transfer Enhancement in Nano-Fluids Suspensions: Possible Mechanisms and Explanations, International Journal of Heat and Mass Transfer, vol. 48, pp. 2673-2683, 2005.

[13] Vadasz, P., Heat Conduction in Nano-Fluid Suspensions, Journal of Heat Transfer, vol. 128, pp. 465-477, 2006.

[14] Choi, U. S., Enhancing Thermal Conductivity of Fluids with Nanoparticles, in Developments and Applications of Non-Newtonian Flows, eds. D. A. Siginer and H. P. Wang, American Society of Mechanical Engineers, New York, pp. 99-105, 1995.

[15] Maxwell, J. C., A Treatise on Electricity and Magnetism, Clarendon Press, Oxford, UK, 1873.

[16] Das, S. K., Choi, S. U. S., and Patel, H. E., Heat Transfer in Nanofluids-A Review, Heat Transfer Engineering, vol. 27, pp. 3-19, 2006.

[17] Wenhua, Y., France, D. M., Routbort, J. L., and Choi, S. U. S., Review and Comparison of Nanofluid Thermal Conductivity and Heat Transfer Enhancements, Heat Transfer Engineering, vol. 29, pp. 432-460, 2008.

[18] Chandrasekar, M., and Suresh, S., A Review on the Mechanisms of Heat Transport in Nanofluids, Heat Transfer Engineering, vol. 30, pp. 1136-1150, 2009.

[19] Eastman, J. A., Choi, S. U. S., Li, S., Yu, W., and Thompson, L. J., Anomalously Increased Effective Thermal Conductivities of Ethylene Glycol-Based Nanofluids Containing Copper Nanoparticles, Applied Physics Letters, vol. 78, pp. 718-720, 2001.

[20] Lee, S., Choi, S. U. S., Li, S., and Eastman, J. A., Measuring Thermal Conductivity of Fluids Containing Oxide Nanoparticles, Journal of Heat Transfer, vol. 121, pp. 280-289, 1999.

[21] Choi, S. U. S., Zhang, Z. G., Yu, W., Lockwood, F. E., and Grulke, E. A., Anomalous Thermal Conductivity Enhancement in Nanotube Suspensions, Applied Physics Letters, vol. 79, pp. 2252-2254, 2001.

vol. 31 no. 142010 
[22] Keblinski, P., Phillpot, S. R., Choi, S. U. S., and Eastman, J. A., Mechanisms of Heat Flow in Suspensions of NanoSized Particles (Nanofluids), International Journal of Heat and Mass Transfer, vol. 45, pp. 855-863, 2002.

[23] Louge, M., and Chen, X., Heat Transfer Enhancement in Suspensions of Agitated Solids, Part III: Thermophoretic Transport of Nanoparticles in the Diffusion Limit, International Journal of Heat and Mass Transfer, vol. 51, pp. 5130-5143, 2008.

[24] Putnam, S. A., Cahill, D. G., Ash, B. J., and Schadler, L. S., High-Precision Thermal Conductivity Measurements as a Probe of Polymer/Nanoparticle Interfaces, Journal of Applied Physics, vol. 94, pp. 6785-6788, 2003.

[25] Putnam, S. A., Cahill, D. G., Braun, P. V., Ge, Z., and Shimmin, R. G., Thermal Conductivity of Nanoparticle Suspensions, Journal of Applied Physics, vol. 99, pp. 0843081-084308-6, 2006.

[26] Prasher, R., Evans, W., Meakin, P., Fish, J., Phelan, P., and Keblinski, P., Effect of Aggregation on Thermal Conduction in Colloidal Nanofluids, Applied Physics Letters, vol. 89, pp. 143119-1-143119-3, 2006.

[27] Blackwell, J. H., A Transient-Flow Method for Determination of Thermal Constants of Insulating Materials in Bulks, Journal of Applied Physics, vol. 25, pp. 137-144, 1954.

[28] Tavman L. H., Effective Thermal Conductivity of Granular Porous Materials, International Communications in Heat and Mass Transfer, vol. 23, pp. 169-176, 1996.

[29] Tavman L. H., Effective Thermal Conductivity of Isotropic Polymer Composites, International Communications in Heat and Mass Transfer, vol. 25, pp. 723-732, 1998.

[30] Tavman, I. H., and Akinci, H., Transverse Thermal Conductivity of Fiber Reinforced Polymer Composites, International Communications in Heat and Mass Transfer, vol. 27, pp. 253-261, 2000.

[31] TP02, Non-Steady-State Probe for Thermal Conductivity Measurement, Users Manual, Hukeseflux Thermal Sensors, The Netherlands, 2001.

[32] Mikhailov, M. D., and Özisik, M. N., Unified Analysis and Solutions of Heat and Mass Diffusion, John Wiley, New York, 1984.

[33] Özisik, M. N., Heat Conduction, 2nd ed., John Wiley, New York, 1993.

[34] Louge, M., and Chen, X., Heat Transfer Enhancement in Suspensions of Agitated Solids, Part I: Theory, International Journal of Heat and Mass Transfer, vol. 51, pp. 5108-5118, 2008.

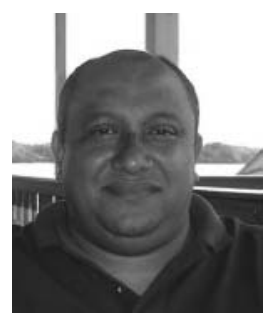

João N. N. Quaresma received his B.Sc. in chemical engineering from the Universidade Federal do Pará in 1988, and his M.Sc. in chemical engineering in 1991 and D.Sc. in mechanical engineering in 1997, both from the Universidade Federal do Rio de Janeiro, Brazil. He joined the School of Chemical Engineering at Universidade Federal do Pará (FEQ/UFPA) in 1991, where he is currently an associate professor, and served for two years as graduate coordinator for the M.Sc. program in chemical engineering. He is the author of more than 80 technical papers in major journals and conferences, and supervisor of 13 Ph.D. and M.Sc. theses. His research interests include the modeling and simulation of non-Newtonian fluid flow, as well as the hybrid solution methodologies in the field of heat and fluid flow. He is the recipient of the Clara Martins Pandolfo Award, given by the Chemistry Council in the State of Pará in 2007. He was a member of the Thermal Sciences Committee of ABCM-Brazilian Society of Mechanical Sciences and Engineering (a sister society of ASME), elected for the period 2004-2008. He is also an $1 \mathrm{C}$ researcher of CNPq, a sponsoring agency in Brazil.

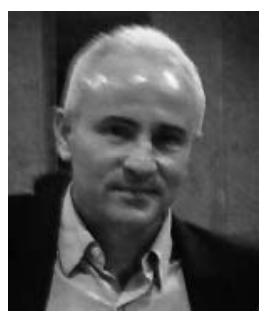

Emanuel N. Macêdo received his B.Sc. in chemical engineering from the Universidade Federal do Pará in 1993 and D.Sc. in mechanical engineering in 1998 from the Universidade Federal do Rio de Janeiro, Brazil. He worked in the Mechanical Engineering Department at the Universidade Federal do Pará as a postdoctoral researcher in the period from 1998 to 2002, and joined the School of Chemical Engineering also at Universidade Federal do Pará (FEQ/UFPA) in 2002, where currently he is an associate professor and the head of the Laboratory of Processes Simulation. His main research area involves the development of hybrid analytical-numerical approaches in the field of heat and fluid flow involving non-Newtonian fluids and combustion processes. Currently, he is the graduate coordinator for the D.Sc. program in natural resources engineering for the Amazon region.

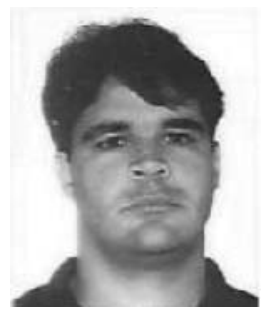

Henrique M. da Fonseca obtained his B.S. in mechanical engineering from the Federal University of Rio de Janeiro (UFRJ) in 2004. After obtaining his M.S. in mechanical engineering in 2007 from the same university, he started his Ph.D. as a joint degree between UFRJ and the Ecole de Mines d'Albi Carmaux, in France, where his research subject is the evaluation of the thermal signature in microfluidic reactors from biological medium submitted to a toxicological stress, under the supervision of Prof. O. Fudym and Prof. H. R. B. Orlande. He is the co-author of more than 10 papers in major journals and conferences.

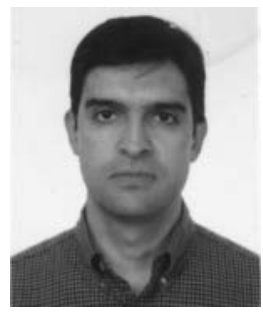

Helcio R. B. Orlande obtained his B.S. in mechanical engineering from the Federal University of Rio de Janeiro (UFRJ) in 1987 and his M.S. in mechanical engineering from the same university in 1989. After obtaining his Ph.D. in mechanical engineering in 1993 from North Carolina State University, he joined the Department of Mechanical Engineering of UFRJ, where he was the department head during 2006 and 2007. His research areas of interest include the solution of inverse heat and mass transfer problems, as well as the use of numerical, analytical, and hybrid numerical-analytical methods of solution of direct heat and mass transfer problems. He is the co-author of one book and more than 160 papers in major journals and conferences. He has been elected distinguished professor by the mechanical engineering classes of UFRJ in 1996 and from 1999 to 2006. He is the recipient of the Young Scientist Award given by the state of Rio de Janeiro in 2000, and of the State Scientist Award given by the state of Rio de Janeiro in 2002, 2004, and 2008. He was the secretary of the Thermal Sciences Committee of ABCM-Brazilian Society of Mechanical Sciences and Engineering (a sister-society of ASME), elected for the period 2005-2006. He is an associate editor of Heat Transfer Engineering, Inverse Problems in Science and Engineering, and High Temperatures-High Pressures. 


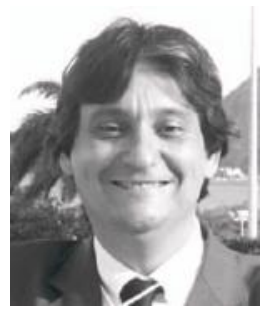

Renato M. Cotta received his B.Sc. in mechani$\mathrm{cal} /$ nuclear engineering from the Universidade Federal do Rio de Janeiro in 1981, and his Ph.D. in mechanical and aerospace engineering from North Carolina State University, USA, in 1985. In 1987 he joined the Mechanical Engineering Department at POLI/COPPE/UFRJ, Universidade Federal do Rio de Janeiro, where he became a full professor in 1997. He is the author of around 370 technical papers and 4 books. He serves in the honorary editorial boards of the International Journal of Heat and Mass Transfer and International Communications in Heat and Mass Transfer, International Journal of Thermal Sciences, International Journal of Numerical Methods in
Heat and Fluid Flow, High Temperatures-High Pressures, Computational Thermal Sciences, Waste and Biomass Valorization, and Advances in Heat Transfer Series, CMP. He was also the editor-in-chief for the international journal Hybrid Methods in Engineering. Prof. Cotta contributed as elected president to the Brazilian Association of Mechanical Sciences, ABCM, in 2000-2001, to the Scientific Council of the International Centre for Heat and Mass Transfer (ICHMT) since 1993, to the Executive Committee of the ICHMT since 2006, as head of the Heat Transmission and Technology Laboratory since 1994, and as head of the Center for Analysis and Simulations on Environmental Engineering, CASEE, a research consortium involving EPRI (USA), Tetra Tech (USA), and COPPE/UFRJ (Brazil), since 2001. He is an elected member of the National Honor Society of Phi Kappa Phi, USA (1984). 